\title{
As estratégias de enfrentamento do COVID-19 pela atenção básica
}

\author{
COVID-19 coping strategies for primary care
}

\author{
Estrategias de afrontamiento de COVID-19 para la atención primaria
}

Davi Ramos do Nascimento ${ }^{1 *}$, Michela Macedo Lima Costa ${ }^{1}$, Lucas Fernandes Soares Matos ${ }^{1}$, Julia Trancoso de Souza ${ }^{2}$, Lorem lasmin Pereira Mendes ${ }^{2}$, Caio Ferreira Leite ${ }^{3}$, Gabriela Pires dos Santos ${ }^{3}$, John Junior Sanzetenea Sanabria ${ }^{4}$, Tauany Maria de Cássia Souza ${ }^{4}$, Loren Mayara Martins Magalhães ${ }^{5}$.

\section{RESUMO}

Objetivo: Analisar quais foram as estratégias de promoção da saúde utilizadas pela Atenção Básica no combate contra o COVID-19. Revisão bibliográfica: A Atenção Primária a Saúde (APS) se estabeleceu como um dos componentes mais abrangente na atenção à saúde, tendo em vista sua longitudinalidade para o cuidado que se traduz em uma melhora na qualidade de vida da população. Entretanto, desde o início da pandemia pelo COVID-19, declarada em 11 de março de 2020, o sistema de saúde público brasileiro vem sendo posto em prova, necessitando de medidas inovadoras que garantam a integralidade da saúde da população, tendo a APS, um papel fundamental no processo de impedimento da progressão dessa pandemia. Considerações finais: Visto o perfil desafiador da pandemia pelo COVID-19, ações como a vigilância em saúde, suporte aos pacientes com COVID-19, apoio social aos grupos vulneráveis e continuidade das ações próprias da APS se tornaram fundamentais para o combate contra a pandemia. Assim sendo, este estudo apresenta fundamentação teórica sobre as principais medidas utilizadas pela atenção primária no combate à pandemia.

Palavras-chave: Atenção básica, Pandemia, Estratégias de saúde, COVID-19.

\begin{abstract}
Objective: To analyze the health promotion strategies used by Primary Care in the fight against COVID-19. Bibliographic review: Primary health care $(\mathrm{PHC})$ has established itself as one of the most comprehensive components in health care, in view of its longitudinally for care, which translates into an improvement in the population's quality of life. However, since the beginning of the COVID-19 pandemic, declared on March 11, 2020 , the Brazilian public health system has been put to the test, requiring innovative measures to ensure the integrality of the population's health, with $\mathrm{PHC}$, a fundamental role in the process of preventing the progression of this pandemic. Final considerations: Given the challenging profile of the pandemic by COVID-19, actions such as health surveillance, support for patients with COVID-19, social support for vulnerable groups, and continuity of PHC's own actions become fundamental to the fight against a pandemic. Therefore, this study presents the theoretical foundation on the main measures used by primary care to fight the pandemic.
\end{abstract}

Keywords: Primary care, Pandemic, Health strategies, COVID-19.

\section{RESUMEN}

Objetivo: Analizar las estrategias de promoción de la salud utilizadas por Atención Primaria en la lucha contra el COVID-19. Revisión bibliográfica: La atención primaria de salud (APS) se ha consolidado como uno de los componentes más integrales de la atención de la salud, dada su longitudinalidad asistencial, que se traduce en una mejora en la calidad de vida de la población. Sin embargo, desde el inicio de la pandemia COVID-19, declarada el 11 de marzo de 2020, el sistema de salud pública brasileño ha sido puesto a prueba, requiriendo medidas innovadoras para asegurar la integralidad de la salud de la población, con la APS, papel

\footnotetext{
${ }^{1}$ Faculdade Santo Agostinho (FASA), Vitória da Conquista - BA. *E-mail: davi.ramos2009@hotmail.com

2 Centro Universitário de Tecnologia e Ciências (UNIFTC), Salvador - BA.

3 Universidade Salvador (UNIFACS), Salvador - BA.

4 Faculdades Integradas Padrão Guanambi (FIPGuanambi), Guanambi - BA.

5 Universidade Estadual de Montes Claros (Unimontes), Montes Claros - MG.
} 
fundamental en la el proceso de prevención de la progresión de esta pandemia. Consideraciones finales: Dado el perfil desafiante de la pandemia por COVID-19, acciones como la vigilancia de la salud, el apoyo a los pacientes con COVID-19, el apoyo social a los grupos vulnerables y la continuidad de las acciones propias de la APS se vuelven fundamentales para la lucha contra una pandemia. Por tanto, este estudio presenta el fundamento teórico sobre las principales medidas que utiliza la atención primaria para combatir la pandemia.

Palabras clave: Atención primaria, Pandemia, Estrategias de salud, COVID-19.

\section{INTRODUÇÃO}

Em dezembro de 2019, o Coronavírus do tipo 2, causador da Síndrome respiratória aguda grave (SARSCoV-2) foi detectado pela primeira vez na cidade de Wuhan, província de Hubei na China como o causador de um grupo de casos de pneumonia. Desde então, o novo vírus se estabeleceu como uma epidemia em todo território chinês, que posteriormente se propagou por todo o mundo (MCINTOSH K, 2021).

No dia 12 de março de 2020, a Organização Mundial da Saúde (OMS) declarou como pandemia a doença causada pelo novo coronavírus 2019 (COVID-19). Por conseguinte, a assistência prestada aos pacientes infectados precisou dar conta da nova demanda e das diferentes necessidades impostas por tal. Nesse cenário, recomendou-se que todos os países aumentassem o seu nível de preparação e fortalecessem os sistemas de alerta para identificar, gerir e cuidar de novos casos de COVID-19, além de comunicar os riscos. (LI Q, et al., 2020).

Por se tratar de uma nova doença, não se tinham planos estratégicos para o seu combate. Sendo assim, a partir de recomendações da OMS, Ministério da Saúde do Brasil, Center for Disease Control and Prevention (CDC) e outras organizações nacionais e internacionais, viu-se possível a aplicação de planos de continência e outras ferramentas da influenza, devida às suas semelhanças clínicas e epidemiológicas com o COVID-19 (FREITAS ARR, et al., 2020).

A partir do Plano de Influenza Pandêmico, desenvolvido pelo Departamento de Saúde e Serviços Humanos (Department of Health and Human Services) dos Estados Unidos, a pandemia pelo Covid-19 foi inserida no Quadro de Avaliação da Gravidade Pandêmica (Pandemic Severity Assessment Framework - PSAF). Pela análise de critérios de risco como transmissibilidade e gravidade clínica, o COVID-19 se apresentou como uma enfermidade de grande seriedade (REED C, et al., 2020).

Em diversos países do mundo as respostas primárias no combate contra a pandemia se concentraram na adoção de medidas de isolamento de casos e contatos, distanciamento social da população e a ampliação dos insumos da terapia intensiva, especialmente os respiradores pulmonares, para que assim, a nova demanda de pacientes graves fosse adequadamente manejada. Por mais, surgiu a necessidade de proporcionar assistência aos pacientes infectados nas diferentes fases da doença, englobando todo o seu espectro de gravidade. Para tal, a Atenção Primária a Saúde (APS) se concretizou como peça fundamental na garantia da integridade da saúde desses pacientes, em uma linha de cuidado que envolve desde o acompanhamento de casos leves em isolamento domiciliar, com instruções para o manejo de sintomas, até o reconhecimento precoce de sinais de alerta (LEITE IC, et al., 2020).

Conforme Nedel FB (2020), ainda que as novas demandas da pandemia requeiram métodos de intervenção inovadores, essas exigem validações de princípios fundadores da APS, como a integralidade da atenção, o trabalho em equipe multiprofissional e a prática clínica e comunitária organizada sobre uma base territorial.

A partir da adesão dos canais de teleconsultas, trabalho de educação em saúde, planejamento baseado em dados, alocação de recursos financeiros e estratégias de ação, a APS se mostrou um potente agente na redução das iniquidades em saúde. Por mais, uma APS forte, organizada e com uma equipe qualificada pode contribuir para reduzir as morbidades na população, com impacto direto no auxílio do controle da pandemia pelo COVID-19 (SARTI TD, et al., 2020).

Partindo desses pressupostos, considera-se que a reorganização das estratégias de promoção da saúde pela atenção básica é uma medida de grande importância para a saúde pública, pois é capaz de reduzir substancialmente as taxas de mortalidade e morbidade (SOUZA DO, 2020). 
Diante do exposto, o presente estudo possui como objetivo compreender quais foram as estratégias de promoção da saúde utilizadas pela Atenção Básica no combate contra o COVID-19.

\section{REVISÃO BIBLIOGRÁGICA}

No dia 26 de fevereiro de 2020, o primeiro caso de COVID-19 foi confirmado no Brasil, um homem de 61 anos que residia na capital paulista e que havia feito uma viagem para a Itália dias atrás (RODRIGUEZMORALES, et al., 2020). Desde o primeiro caso confirmado, ocorreu o crescimento exponencial dos infectados, até o início da vacinação, sendo que, na data de 18/09/2021, cerca de 20.080.219 de casos da doença foram confirmados e 589.573 óbitos foram registrados (MINISTÉRIO DA SAÚDE, 2021).

Segundo Croda JHR (2020), o imediatismo na resposta da Secretaria de Vigilância em Saúde foi evidente. Para tal, os Pontos Focais Nacionais do Regulamento Sanitário Internacional da OMS foram acionados no início de janeiro, fortalecendo a vigilância e resposta às emergências em saúde pública. Já no final desse mês, foi efetivado o Centro de Operações de Emergência do Ministério da Saúde, para harmonização, planejamento e organização das atividades com os atores envolvidos com o monitoramento internacional e, além disso, foi ativado o plano de contingência, sistematizando medidas de resposta para infecção humana pelo novo vírus. Por fim, no dia 3 de fevereiro de 2020, a epidemia foi declarada Emergência em Saúde Pública de Importância Nacional.

Como consequência da sua consolidação e visível repercussão como um dos maiores desafios sanitários do século, debates acerca dos conceitos e modelos de atenção à saúde que devem ser adotados para cumprir critérios que atendam as diretrizes e princípios do Sistema Único de Saúde (SUS) se tornaram presentes. Tais debates levam em conta âmbitos acadêmicos, econômicos, políticos e sociais, que visam a garantia da integralidade da saúde da população brasileira (CABRAL ERM, et al., 2020).

A partir da Lei № 8080, o SUS se estabeleceu como um dos maiores e mais complexos sistemas de saúde pública do mundo. Com as suas reformas e desenvolvimento ao longo dos anos, a organização da Atenção Primária à Saúde (APS) e progressiva implementação da Estratégia Saúde da Família (ESF) fez com que esse sistema garantisse acesso integral e universal para a população brasileira. Com a atenção ao primeiro contato, a hierarquização dos níveis de atenção à saúde, regionalização a partir de bases populacionais e prestação de serviços ambulatoriais que podem resolver cerca de $80 \%$ de todas as queixas de saúde da população, a atenção básica se tornou uma das principais e mais eficientes estratégias de saúde pública brasileira (PORTELA GZ, 2017; ALMEIDA PF, 2011).

A atenção integral à saúde, e não somente os cuidados assistenciais, passaram a ser um direito de todos os brasileiros, desde o nascimento, até a velhice, com foco na qualidade de vida pela gestão descentralizada, participação da comunidade e pela adoção de estratégias que promovam tal atenção. O principal colaborador para a integralidade desses atendimentos é a APS, que, através da coordenação do cuidado, acessibilidade e longitudinalidade, garantem um vínculo de longa duração com os usuários da atenção primária (PORTELA GZ, 2017).

A APS, a partir da aprovação da Política Nacional de Atenção Básica (PNAB) em 2006 pelo Ministério da saúde, revisado em 2011 e atualizado em 2017, tem como função contribuir para reorganizar o modelo assistencial de acordo com os princípios do SUS, firmando um novo processo de atuação nas unidades básicas de saúde, com o compartilhamento de responsabilidades entre os serviços de saúde e a população. Isto posto, a APS se revelou como o componente mais abrangente na atenção à saúde, tendo em vista sua centralidade para o cuidado que se fundamenta em princípios, diretrizes e eixos para a produção do cuidado (FERREIRA JS e LADEIA LEG, 2018).

A pandemia de 2020, após sua visível repercussão sanitária, vem pondo em prova os diversos serviços de saúde pública espalhados pelo mundo. Por meio um imenso estresse pela nova demanda de pacientes e cobrança de respostas em uma velocidade nunca antes prevista, foi demandado do SUS uma maior eficiência e capilaridade, para que assim, esse consiga ainda mais reduzir desigualdades e vulnerabilidades da população. Dessa forma, vê-se importância da reinvenção SUS em momentos de crise, garantindo e ampliando o conceito de saúde (BOUSQUAT A, et al., 2021). 
Um dos fatores agravantes, em relação ao SUS, é que esse vem sofrendo problemas crônicos de subfinanciamento e falhas administrativas que agudizaram com o início da pandemia. Em vista disso, estratégias conflitantes de combate à pandemia entre o governo federal e os demais entes federativos geraram uma descoordenação nacional acerca de planos administrativos, reforçando a desigualdade estrutural causada pela pandemia. Além da desordem administrativa, os recursos habitualmente insuficientes destinados ao SUS, estão ainda mais diminuídos para o enfrentamento dessa grave crise sanitária (FUNCIA FR, 2019).

Com a falta de medidas de controle curativas, preventivas, como as vacinas, e devido à alta taxa de transmissibilidade da infecção evidenciado pelo PSAF, as intervenções mais eficazes para o controle da pandemia foram medidas como distanciamento social, vigilância e isolamento dos casos, adoção da obrigatoriedade do uso de máscaras em ambientes fechados e a recomendação para a higienização constante das mãos e superfícies com produtos à base de álcool. Tais recomendações foram cada vez mais estimuladas pela Organização Mundial da Saúde para a minimização dos efeitos da pandemia, possibilitando que o sistema de saúde não colapsasse nesse período (KRATZEL A, et al., 2020).

Diante desse quadro, a resposta sanitária primária se concentrou na ampliação dos serviços hospitalares, como serviços de terapia intensiva e na aquisição de respiradores pulmonares. Porém, além dessas medidas, viu-se a necessidade de ampliar os serviços prestados pela atenção primária, criando novos fluxos de atendimento para atender os pacientes com a suspeita clínica da doença (MEDINA MG, et al., 2020).

Nesse sentido, para corroborar com tais medidas, uma maior cobertura da APS é necessária para mitigar os efeitos da pandemia através do potencial deste modelo de atenção. Contudo, mesmo com uma extensa rede de atenção básica distribuída em parte do território brasileiro, essa apresenta problemas crônicos de subfinanciamento, gestão, provisão de profissionais e estruturação dos serviços. A despeito disso, a atenção primária brasileira tem conseguido resultados positivos em toda a sua abrangência, resultando em um destaque positivo no âmbito internacional (SARTI TD, et al., 2020).

Ao implementar os atributos da atenção básica, como o acesso ao primeiro contato, a longitudinalidade, integralidade, coordenação do cuidado e, em especial, a competência cultural e comunitária, a rede básica se mostra como o modelo mais adequado para propor medidas preventivas por seus atributos de responsabilidade territorial (PORTELA GZ, 2017).

Segundo Medina MG (2020), o planejamento logístico, capacitação e organização da APS permite que essa se disponha em quatro eixos distintos, que sistematizam a sua atuação no combate contra a pandemia, sendo eles a vigilância em saúde nos territórios, atenção aos usuários com COVID-19, suporte social a grupos vulneráveis e a continuidade das ações próprias da APS. Tais eixos estabelecem a necessidade de lidar simultaneamente com o papel central de controle da pandemia e assegurar que as ações já previstas para a APS não sejam abandonadas.

A vigilância em saúde, atuação da APS em conjunto com a vigilância epidemiológica e vigilância sanitária, tem como atribuições o gerenciamento de risco no território adscrito, gerando um fluxo de informações para o aprimoramento das intervenções, notificação, detecção e acompanhamento dos pacientes suspeitos de COVID-19, desenvolvimento de atividades educativas que incentivem a higienização e saúde coletiva, reforçar a força laboral da APS, podendo envolver estudantes, difusão de informações para a comunidade sobre prevenção de doenças, promoção do isolamento domiciliar nos casos suspeito, e por fim, a realização da testagem da população adscrita (SALES CMM, et al., 2020).

Como peça chave nesse controle de vigilância, os agentes comunitários de saúde (ACS), promotores da competência cultural, orientação comunitária e construção de vínculo, se mostraram como grandes incentivadores do isolamento social. Os ACS possibilitam a mobilização de lideranças e recursos locais com ampla divulgação de informações, sendo responsáreis pelo processo de apoio às equipes de saúde, utilização da telesaúde e educação continuada (MACIEL FBM, et al., 2020).

Com o aumento da demanda nas unidades, a implementação de novos fluxos de atendimento se tornou uma medida eficiente. A partir dessa, a identificação precoce, principalmente em comunidades vulneráveis, pode reduzir a velocidade de transmissão do COVID-19, com a recomendação do isolamento de pessoas 
sintomáticas e oligossintomáticas. Nesse sentido, a OMS propôs a implementação de canais de teleatendimento 24 horas, que, a partir de um treinamento específico, os atendentes se tornam capacitados para identificação de casos leves e graves e para realizar orientação individualizadas para os pacientes. Além disso, a OMS ressalta a importância da coordenação entre entes governamentais para melhor fluxo de recursos (WORLD HEALTH ORGANIZATION, 2020).

Além dos mecanismos de vigilância, o suporte a população vulnerável como idosos, mulheres grávidas, portadores de diabetes, hipertensão com o mantimento da prestação de serviços essenciais de atenção primária (não relacionados à COVID-19) se mostraram estratégias cruciais no combate contra a pandemia. Para tal, o Núcleos de Apoio à Saúde da Família (NASF) se torna extremamente essencial no cotidiano da atenção primária, sendo responsáveis por atividades com o intuito de promover apoio psicológico à essa população, atividades esportivas, consultas a nutricionistas, entre outros (SAMPAIO J, 2012).

Para a garantia da continuidade dos atendimentos oferecidos pela APS, a adoção da telemedicina se mostrou como ferramenta efetiva, com a redução do contato presencial entre profissionais de saúde e pacientes infectados, com a criação de novos pontos de acesso ao sistema de saúde (WORLD HEALTH ORGANIZATION, 2020). Além da realização das consultas, os aplicativos de comunicação como "WhatsApp" podem ser utilizados para renovação de receitas e a busca por medicamentos, sendo a entrega realizada pelo ACS. Também, as modalidades de teleatendimento (teleconsulta, teleorientação, telerregulação e telemonitoramento) se tornaram ferramentas efetivas a serem utilizadas no cotidiano da unidade (LEITE IC, 2020).

Também, como atribuição da APS, o atendimento aos problemas de saúde de consequência da pandemia deve ser devidamente manejado, sendo eles alcoolismo, agudização de agravos crônicos, transtornos mentais, violência doméstica e outros. Vale lembrar que tais agravos serão uma demanda além da já atendida por essas unidades, exigindo uma preparação para tal (SARTI TD, et al., 2020).

Como já exposto, o grande subfinanciamento presente da APS brasileira impede o seu desenvolvimento. Nesse sentido, a crônica falta de atenção dos gestores em relação a saúde brasileira se tornou mais evidente na crise da pandemia, se refletindo na capacidade de atuação da atenção básica em tal situação. A exemplo, a falta de Equipamentos de Proteção Individual (EPI), baixa disponibilidade de computadores e acesso internet que restringem o alcance e a atuação das unidades no período da pandemia. Com um maior fortalecimento da APS, uma maior cobertura das equipes seria alcançada, viabilizando a Estratégia de Saúde da Família (ESF) no âmbito da Atenção Primária (CABRAL ERM, et al., 2020).

Nesse sentido, a proteção dos profissionais de saúde frente ao novo vírus é de extrema importância. A partir da aquisição de EPI, como protetores faciais, máscaras cirúrgicas, gorros, luvas, máscaras N95 além de outros insumos, tais profissionais são capazes de preservar a própria saúde além de evitar que passem a ser um novo foco de infecção (MUNSTER VJ, et al., 2020).

A proteção da população brasileira se viu posta em risco a partir da falta de EPI. No início da pandemia, esses tiveram que ser racionados, pois com a nova demanda mundial, a sua disponibilidade se viu ameaçada. A falta de insumos reverberou em diversos setores da saúde, desde o atendimento ambulatorial na atenção primária, até profissionais em ambiente de terapia intensiva. Quando acessíveis, esses eram disponibilizados aos profissionais com o devido monitoramento, para um controle adequado que visava diminuir os desperdícios (RODRIGUES NH e SILVA LGA, 2020).

Mesmo como racionamento desses equipamentos, pode-se observar a má utilização dos EPIs, como máscaras fora do rosto, mãos contaminadas em contato com o equipamento, e erro no descarte. Nesse sentido, o treinamento dos profissionais de saúde para a utilização dos equipamentos de segurança se viu necessária e eficaz. Além disso, alternativas que visaram amenizar a carência de EPI foram elaboradas, como a utilização de máscaras de pano para a população que não tiveram acesso à mascas N95 (TEIXEIRA CFZ, et al., 2020).

No dia 17 de janeiro de 2021, após a Agência Nacional de Vigilância Sanitária autorizar o uso emergencial de duas vacinas no Brasil, a enfermeira Mônica Calazans foi a primeira pessoa a ser imunizada fora de um 
estudo clínico. Desde o início do processo de vacinação no território brasileiro, as expectativas de um desfecho eminente para a pandemia se tornaram realidade. Porém, a gestão da vacinação no Brasil foi marcada por diversas dificuldades, atrasando o processo de imunização da população. Divergências sobre questões relacionadas à vacina entre diferentes entes políticos, se tornaram alvo de grandes discussões acerca da sua eficácia. Como promoção desses discursões, parte de governantes brasileiros utilizaram como método de alienação notícias falsas acerca da imunização, polarizando uma questão de saúde pública, ações essas que vão de contra com as proporcionas na atenção primária (CASTRO R, 2020).

Apesar das divergências políticas impostas a vacinação, essa vêm demonstrando grande eficácia para a prevenção de quadros clínicos graves, devendo ser cada vez mais estimulada. Para o comprimento da tarefa de vacinar a população de risco, o Programa Nacional de Imunização (PNI) se mostrou de tremenda eficácia e necessidade. A partir da sua estruturação com campanhas de vacinação em massa e elevadas coberturas vacinais, essa estratégia auxilia na diminuição da circulação de agentes infecciosos nas comunidades, melhorando a qualidade de vida da população (DOMINGUES CMAS, 2021).

Por fim, vê-se a necessidade do desenvolvimento de tecnologias de enfrentamento das iniquidades, uma abordagem mais territorialidade, comunitária, domiciliar e com a necessidade de uma APS forte e integral, em toda a sua potencialidade no combate contra o COVID-19. Tal abordagem é de indubitável importância para que assim, futuras enfermidades sejam melhor manejadas pelas estratégias aqui revisadas.

\section{CONSIDERAÇÕES FINAIS}

A pandemia pelo COVID-19 se mostrou um problema muito grave e que gerou consequências absolutas na saúde da população. Nesta perspectiva, a promoção e intensificação de estratégias para um combate inteligente contra a pandemia foi de extrema necessidade. Assim sendo, ações como a vigilância em saúde, suporte aos pacientes com COVID-19, apoio social aos grupos vulneráveis e continuidade das ações próprias da APS, baseadas em abordagem mais territorialidade, comunitária e domiciliar se tornaram fundamentais nesse momento de crise. Para possibilitar e viabilizar tais estratégias, é necessário um conjunto investimentos e ações administrativas governamentais planejadas, que, baseadas em constructos científicos como esse, podem gerar grandes repercussões positivas na saúde da população.

\section{REFERÊNCIAS}

1. ALMEIDA PF, et al. Fortalecimento da atenção primária à saúde: estratégia para potencializar a coordenação dos cuidados. Revista Panamericana de Salud Pública, 2011; 29: 84-95.

2. BOUSQUAT, A. et al. Pandemia de covid-19: o SUS mais necessário do que nunca. Revista USP, $2021 ; 128$ : 13-26.

3. CABRAL ERM, et al. Contribuições e desafios da Atenção Primária à Saúde frente à pandemia de COVID-19. InterAm J Med Health, 2020; 3: e202003012

4. CASTRO R. Vacinas contra a Covid-19: o fim da pandemia 2019. Physis: Revista de Saúde Coletiva. 2020; 31(1): e310100.

5. CRODA JHR. Resposta imediata da Vigilância em Saúde à epidemia da COVID-19. Epidemiologia e Serviços de Saúde, 2020; 29(1): e2020002.

6. DOMINGUES CMAS. Desafios para a realização da campanha de vacinação contra a COVID-19 no Brasil. Cadernos de Saúde Pública, 2021; 37(1): e00344620.

7. FERREIRA JS, LADEIA LEG. As Implicações da Política Nacional de Atenção Básica (PNAB) na Dinâmica dos Serviços de Saúde. Id On Line Revista de Psicologia, 2018; 12(4): 681-695.

8. FREITAS ARR, et al. Análise da gravidade da pandemia de Covid-19. Epidemiologia e Serviços de Saúde, 2020; 29(2): e2020119.

9. FUNCIA FR. Subfinanciamento e orçamento federal do SUS: referências preliminares para a alocação adicional de recursos. Ciência \& Saúde Coletiva, 2019; 24(12): 4405-4415.

10. LAVRAS C, et al. Atenção primária à saúde e a organização de redes regionais de atenção à saúde no Brasil. Saúde e Sociedade, $2011 ; 20(4): 867-874$.

11. LEITE IC, et al. O papel da atenção primária na rede de atenção à saúde no Brasil: limites e possibilidades no enfrentamento da COVID-19. Cad. Saúde Pública, Rio de Janeiro, 2020; 36(6): e00104120. 
12. LI Q, et al. Early transmission dynamics in Wuhan, China, of novel coronavirus-infected pneumonia. N Engl J Med, 2020; 382: 1199-207.

13. MACIEL FBM, et al. Agente comunitário de saúde: reflexões sobre o processo de trabalho em saúde em tempos de pandemia de Covid-19. Ciência \& Saúde Coletiva, 2020; 25(2): 4185-4195.

14. MCINTOSH K. 2021. In: Coronavirus disease 2019 (COVID-19). UptoDate. Disponível em: https://www.uptodate.com/contents/126981. Acesso em: 17 setembro. 2021

15. MEDINA MG, et al. Atenção primária à saúde em tempos de COVID-19: o que fazer?. Cad. Saúde Pública, 2020; 36(8): e00149720.

16. MINISTÉRIO DA SAÚDE. COVID19, Painel Coronavírus 2021. In: CORONAVIRUS BRASIL. Disponível em: https://covid.saude.gov.br/. Acessado em: 18 de setembro de 2021.

17. MUNSTER VJ, et al. A novel coronavirus emerging in china- key questions for impact assessment. N Eng J Med, 2020; 382: 692-4.

18. NEDEL FB. Enfrentando a COVID-19: aps forte agora mais que nunca!. Aps em Revista, 2020; 2(1): $11-16,15$.

19. PORTELA GZ. Atenção Primária à Saúde: um ensaio sobre conceitos aplicados aos estudos nacionais. Physis: Revista de Saúde Coletiva, 2017; 27(2): 255-276.

20. REED C, et al. Novel framework for assessing epidemiologic effects of influenza epidemics and pandemics. Emerg Infec, 2013; 19(1): 85-91.

21. RODRIGUES NH, SILVA LGA. Gestão da pandemia coronavírus em um hospital: relato de experiência. Journal Of Nursing And Health, 2020; 10(4): 1-9.

22. RODRIGUEZ-MORALES, et al. COVID-19 in Latin America: the implications of the first confirmed case in brazil. Travel Medicine And Infectious Disease, 2020; 35: 101613.

23. SALES CMM, et al. Vigilância em saúde da COVID-19 no Brasil: investigação de contatos pela atenção primária em saúde como estratégia de proteção comunitária. Epidemiologia e Serviços de Saúde, 2020; 29(4): 1-6.

24. SAMPAIO J, et al. O NASF Como Dispositivo da Gestão: limites e possibilidades. Revista Brasileira de Ciências da Saúde, 2012; 16(3): 317-324.

25. SARTI TD, et al. Qual o papel da Atenção Primária à Saúde diante da pandemia provocada pela COVID-19. Epidemiol Serv Saúde, 2020; 29(2): e2020166.

26. SOUZA DO. A pandemia de COVID-19 para além das Ciências da Saúde: reflexões sobre sua determinação social. Ciência \& Saúde Coletiva, 2020; 25: 2469-2477.

27. TEIXEIRA CFZ, et al. A saúde dos profissionais de saúde no enfrentamento da pandemia de Covid-19. Ciência \& Saúde Coletiva, 2020; 25(9): 3465-3474.

28. WORLD HEALTH ORGANIZATION (WHO). COVID-19 strategy update - 14 April 2020. Geneva: World Health Organization, 2020; 18 p. Disponível em: https://www.who.int/publications/i/item/covid-19-strategy-update---14-april2020. Acessado em: 18 de setembro de 2021.

29. KRATZEL A, et al. Inactivation of Severe Acute Respiratory Syndrome Coronavirus 2 by WHO-Recommended Hand Rub Formulations and Alcohols. Emerg Infect Dis, 2020; 26(7): 1592-1595. 